\title{
Niveles de eficiencia de las policlínicas de Matanzas, Cuba, según el método de análisis envolvente de datos
}

\author{
Anai García Fariñas, ${ }^{1}$ Zoe Sánchez Delgado, ${ }^{2}$ Magalys Chaviano Moreno ${ }^{2}$ \\ y Miriam Muñiz Cepero
}

Forma de citar

García Fariñas A, Sánchez Delgado Z, Chaviano Moreno M, Muñiz Cepero M. Niveles de eficiencia de las policlínicas de Matanzas, Cuba, según el método de análisis envolvente de datos. Rev Panam Salud Publica. 2007;22(2):100-9.

RESUMEN Objetivos. Determinar el índice de eficiencia de todas las policlínicas de la provincia de Matanzas, Cuba, identificar las unidades de mejor práctica y estimar las reservas de eficiencia de cada unidad.

Metodología. Se realizó una investigación descriptiva durante el primer trimestre de 2006 en las 40 policlínicas de la provincia de Matanzas, Cuba. Las policlínicas se agruparon según su nivel de complejidad y el nivel socioeconómico del municipio en el que se ubican. Se consideraron cinco indicadores de resultados y seis de recursos. Para los cálculos se empleó el análisis envolvente de datos y se asumieron el modelo de maximización de resultados y los supuestos de rendimientos constantes y variables a escala.

Resultados. La eficiencia relativa de las unidades estudiadas fue alta, con un valor medio de 0,95 $\pm 0,11$. Once $(27,5 \%)$ de las policlinicas estudiadas fueron ineficientes $(0,77 \pm 0,12)$. Las principales reservas para elevar la eficiencia fueron aumentar la detección de los casos de tuberculosis en la comunidad, reducir el índice de mortalidad infantil evitable y ampliar la cobertura de inmunización. En las 11 policlínicas ineficientes había holgura de recursos en al menos un indicador.

Conclusiones. La identificación de policlínicas de referencia permitió aportar elementos organizacionales que podrían contribuir a mejorar la eficiencia de las unidades deficientes. Debe revisarse el proceso de asignación de recursos para evitar el exceso de recursos innecesarios como vía para aumentar la eficiencia.

Palabras clave Investigación sobre servicios de salud, eficiencia, atención primaria de salud, Cuba.

1 Instituto Nacional de Higiene, Epidemiología y Microbiología, Departamento de Salud Pública, Ciudad de La Habana, Cuba. La correspondencia se debe dirigir a Anai García Fariñas, Instituto Nacional de Higiene, Epidemiología y Microbiología, Infanta No. 1158 entre Clavel y Llinás, Centro Habana, CP 10300, Ciudad de La Habana, Cuba. Correo electrónico: anaigf@yahoo.es

2 Dirección Provincial de Salud, Matanzas, Cuba.
La demanda de servicios de salud aumenta cada vez más en todo el mundo. Aunque los recursos destinados al gasto público en salud también se han incrementado, todavía son insuficientes en muchos países y a los gobiernos no les queda otra alternativa que asignar los recursos de acuerdo con un estricto principio de prioridad. Este proceso de asignación implica sacrificios, pues al destinar un recurso para un fin, otros objetivos pueden quedar sin la cobertura necesaria. Ante esta situación, los economistas han se- 
ñalado que el mejor criterio de priorización es emplear los recursos existentes con la mayor eficiencia posible (1).

Según las ciencias económicas (2), la eficiencia se define como la relación entre los resultados obtenidos en una actividad dada y los recursos utilizados; se es eficiente cuando los objetivos fijados se alcanzan sin malgastar recursos. La mayor eficiencia se logra con una mejor relación recursos-resultados, por lo que dependerá directamente de la cantidad y calidad de los resultados e inversamente del volumen de recursos empleados.

En el sector de la salud, eficiencia significa alcanzar los mejores resultados con los recursos disponibles, para lo cual es necesario conocer los procesos técnicos que se deben abordar y las vías para lograrlo. En otras palabras, un sistema de salud es eficiente cuando es capaz de brindar un producto sanitario aceptable para la sociedad con el menor uso de recursos.

Uno de los métodos empleados para determinar la eficiencia es el análisis de frontera - ya sea paramétrico o no paramétrico-, que permite comparar los resultados con valores de referencia (3). Para construir las fronteras de producción y de costos, este tipo de análisis se vale de la mejor práctica observada en una muestra de unidades de toma de decisiones. Entre los métodos paramétricos se encuentran: la frontera estocástica, el enfoque de distribución libre y el enfoque de frontera gruesa (thick frontier approach). Entre los métodos no paramétricos se encuentran el análisis envolvente de datos (AED) y el llamado enfoque de casco de libre disponibilidad (free disposal hull, FDH). ${ }^{3}$

Entre los estudios desarrollados sobre el empleo de diferentes técnicas y métodos para la evaluación de la eficiencia en unidades de salud destacan los estudios realizados en Europa (3-5)

\footnotetext{
3 El enfoque FDH es un caso particular del AED, donde los puntos sobre las líneas que conectan los vértices no forman parte de la frontera. Esto tiene como consecuencia que los estimadores obtenidos con FDH son distintos a los obtenidos con AED. El objetivo primario de este método es asegurar que únicamente los desempeños observados afecten a las estimaciones de eficiencia.
}

y los Estados Unidos de América (6). En general, estos estudios demostraron que el AED es el más frecuentemente empleado para analizar la efectividad en organizaciones sanitarias. La referencia teórica de este método se encuentra en un trabajo publicado en 1957 por Farrel y colaboradores (7). Según esta metodología, se construye un índice de eficiencia para unidades caracterizadas por una situación productiva en la que múltiples insumos generan múltiples productos.

En 1978, Charnes, Cooper y Rhodes (8) publicaron el modelo matemático a partir del cual se desarrolló este método. Este modelo se basa en la programación lineal y toma en cuenta todas las variaciones observadas como ineficiencias y no como productos del azar. En este caso, la función objetivo es:

$$
\begin{aligned}
& h_{o} \max =\frac{\sum_{r=1}^{s} \mathrm{u}_{r} \mathrm{y}_{r o}}{\sum_{i=1}^{m} \mathrm{v}_{i} \mathrm{x}_{i o}} \\
& \text { Sujeto a: } \frac{\sum_{r=1}^{s} \mathrm{u}_{r o} \mathrm{y}_{r j}}{\sum_{i=1}^{m} \mathrm{v}_{i o} \mathrm{x}_{i j}} \leq 1
\end{aligned}
$$

Tal que:

$$
\begin{array}{ll}
j=0,1, \ldots n & \\
u_{r 0} \geq 0 ; & r=1, \ldots s \\
v_{i 0} \geq 0 ; & i=1, \ldots m
\end{array}
$$

donde:

$h_{0}$ : índice de eficiencia

$n$ : número de unidades productivas evaluadas

$s$ : número de productos que se introducen en el modelo

$m$ : número total de recursos que se introducen en el modelo

$i$ : subíndice en la iteración de la variable $m$

$r$ : subíndice en la iteración de la variable $s$

$\mathrm{u}_{r}$ : pesos positivo y desconocido atribuidos al producto $r$

$\mathrm{v}_{i}$ : pesos positivo y desconocido atribuidos al recurso $i$

$j$ : unidad productiva analizada $\mathrm{y}_{r j}:$ cantidades conocida y positiva del producto $r$

$\mathrm{x}_{i j}$ : cantidades conocida y positiva del recurso $i$.

Según Gattoufi y colaboradores (9, 10), la mayoría de los autores e investigaciones que han aplicado el AED son de América del Norte, Inglaterra, Australia y Japón, aunque en los últimos 26 años se observa un crecimiento anual de $25,5 \%$ en el número de artículos publicados que emplean este método. Las principales áreas de aplicación son las organizaciones sin fines de lucro, especialmente las dedicadas a brindar servicios de salud.

Los estudios sobre la eficiencia en instituciones de atención primaria de salud (APS) son recientes, aunque se ha observado un franco incremento en los últimos años. La mayoría de los estudios se han realizado en países industrializados, como los Estrados Unidos, el Reino Unido, Australia y España. Hasta la fecha no se cuenta con una gran experiencia al respecto en América Latina, a pesar de que los gobiernos demandan cada vez más evidencias científicas que apoyen la ampliación de los servicios públicos de salud, tanto en cantidad como en tipos de servicios.

La medición de los resultados de los estudios de eficiencia en los servicios de APS es más compleja que en las instituciones hospitalarias debido a la naturaleza multidimensional de su producción y de sus insumos y a la dificultad de modelar el proceso productivo. El AED puede ser muy útil en estas circunstancias por su mayor flexibilidad y su capacidad de respetar las particularidades productivas de cada entidad.

En Cuba, el uso eficiente de los recursos destinados a los servicios de salud ha sido una premisa permanente de las autoridades nacionales. Si bien desde el punto de vista macroeconómico se han logrado niveles elevados de eficiencia (11), la situación es muy diferente cuando el análisis se lleva al nivel microeconómico. Esto quedó reflejado en las conclusiones y recomendaciones de un grupo de expertos que participaron en el Taller sobre Eficien- 
cia en el Sector Salud y Aspectos Macroeconómicos, celebrado en febrero de 1999 en la Ciudad de La Habana, entre las que resalta el reconocimiento de que "... no somos lo suficientemente eficientes y todavía debemos profundizar en las reservas de eficiencia, sobre todo en el subsistema de atención primaria de salud" (12).

Cabe señalar, además, que a partir de la década de 1990 en Cuba ha disminuido la proporción de los gastos destinados a la atención hospitalaria y ha aumentado la destinada a la APS (2). Este cambio se acentuó más después de la puesta en marcha del Programa Revolución, gracias al cual las policlínicas tuvieron una reparación capital, se modernizaron tecnológicamente y se capacitaron sus recursos humanos. En términos económicos, este proceso ha generado un incremento notable de los recursos disponibles en los servicios médicos primarios cubanos. Sin embargo, no existen antecedentes de estudios de eficiencia en estas instituciones, aun cuando existe una acentuada voluntad política para que estos recursos se empleen con una mejor relación recurso-resultado.

Los objetivos del presente trabajo fueron determinar el índice de eficiencia de todas las policlínicas de la provincia de Matanzas, Cuba, identificar las unidades de mejor práctica y estimar las reservas de eficiencia de cada unidad.

\section{MATERIALES Y MÉTODOS}

Se realizó una investigación descriptiva. Se seleccionó el nivel primario de salud y particularmente las policlínicas, debido a que constituyen la base y el núcleo central estratégico del Sistema Nacional de Salud (SNS) de Cuba (13). Se definió como horizonte temporal el primer trimestre de 2006.

El universo de estudio fueron las 40 policlínicas de la provincia de Matanzas, Cuba. Se seleccionaron todas las policlínicas de la provincia para que la información generada fuera de utilidad para la toma de decisiones a ese nivel y porque un mayor número de unidades permitiría identificar policlínicas con diferentes niveles de eficiencia. Se escogió la provincia de Matanzas para realizar la investigación porque se contaba con los recursos humanos y materiales necesarios para recopilar la información primaria y con el apoyo de las autoridades provinciales de salud.

Para facilitar la adecuada comparación de las policlínicas estudiadas, estas se agruparon según el criterio de clasificación de policlínicas de la Dirección Nacional de Servicios Ambulatorios del Ministerio de Salud Pública cubano: tipo 1 o de mayor complejidad (21 policlínicas) y tipo 2 o de menor complejidad (19 policlínicas). Cada grupo se subdividió en subgrupos: el subgrupo A, formado por los municipios de mayor nivel socioeconómico (Matanzas, Varadero, Cárdenas, Colón y Jagüey) y el subgrupo B, por el resto de los municipios. De esta manera, los subgrupos de comparación quedaron compuestos por 11 policlínicas en el subgrupo 1A, 10 policlínicas en el 1B, 8 policlínicas en el 2A y 11 policlínicas en el subgrupo $2 \mathrm{~B}$.

\section{Variables de resultados y de recursos}

Se adoptaron las variables de resultados y de recursos propuestas por un grupo de consenso nacional en un estudio previo. ${ }^{4}$ Se consideró que, en su conjunto, las cinco variables de resultados permitían medir adecuadamente el cumplimiento del objetivo general de las policlínicas como unidades del SNS: mejorar continuamente el estado de salud de la población (14). Estas variables tienen una adecuada sensibilidad y varían de acuerdo con el desempeño de las policlínicas. En ese estudio también se identificaron seis variables de recursos que se corresponden con los

\footnotetext{
4 García Fariñas A. Propuesta de indicadores de resultados y de recursos del policlínico, primera etapa en la medición de la eficiencia de estas unidades (tesis doctoral). Ciudad de La Habana: Instituto Nacional de Higiene, Epidemiología y Microbiología; 2005.
}

resultados a evaluar (cuadro 1). Estas variables se trabajaron bajo la premisa de que no se toman valores negativos, como exige el AED. La información sobre estos indicadores se tomó de los registros oficiales del sistema de información estadística del SNS y se llevó a una hoja de vaciamiento de datos confeccionada al efecto. Para el caso de los indicadores de costos se utilizó la tasa de cambio oficial del Ministerio de Finazas y Precios de la República de Cuba de 1,00 peso cubano $(\mathrm{MN})=1,00$ peso cubano convertible $(\mathrm{CUC})=1,25$ dólar estadounidense.

Para aumentar el poder discriminatorio del método empleado se debe lograr la menor relación posible del producto de las variables con respecto al número de unidades que se comparan. A fin de utilizar el menor número posible de indicadores en cada subgrupo se realizó un análisis de correlación entre las variables de recursos y las de resultados y se tomó solamente una variable de los pares de datos que mostraron un coeficiente de correlación de Spearman ( $r s$ ) mayor de 0,65 (ya que a criterio de los autores, este es un punto de corte que permite afirmar que el grado de asociación entre dos variables es débil o fuerte). Se prefirió este coeficiente de correlación al de Pearson debido a que estas variables no son continuas y al pequeño tamaño de la muestra.

\section{Modelo de optimización}

Para la optimización se seleccionó el modelo de maximización de resultados, ya que es el adecuado cuando los directivos están interesados en un aumento de la productividad sin que necesariamente esto conlleve a la reducción de los recursos en uso (15). En Cuba, la búsqueda de la eficiencia no contempla medidas de recorte presupuestario y, específicamente en el sector salud, en los últimos veinte años se ha observado el aumento permanente de los recursos disponibles. Con la elección de este modelo se pretende aportar nuevos elementos relacionados con la evaluación del uso de recursos. 
CUADRO 1. Indicadores de resultados y recursos empleados en el estudio

\begin{tabular}{|c|c|c|}
\hline Indicador & Descripción & Fuente de información \\
\hline Mortalidad infantil evitable & $\begin{array}{l}\text { Número de muertes en menores de } 1 \text { año por responsabilidad del servicio de } \\
\text { atención primaria de salud entre el total de muertes en menores de } 1 \text { año }\end{array}$ & $\begin{array}{l}\text { Registro de la policlínica del } \\
\text { Programa Materno Infantil }\end{array}$ \\
\hline Índice de bajo peso al nacer & $\begin{array}{l}\text { Total de niños nacidos con un peso menor de } 2500 \mathrm{~g} \text { entre el total de niños } \\
\text { nacidos vivos, expresado en por ciento }\end{array}$ & $\begin{array}{l}\text { Registro de la policlínica del } \\
\text { Programa Materno Infantil }\end{array}$ \\
\hline $\begin{array}{l}\text { Índice de satisfacción de la } \\
\text { población }\end{array}$ & $\begin{array}{l}\text { Número de encuestados que consideraron adecuados todos los aspectos entre } \\
\text { el total de encuestados, expresado en por ciento }\end{array}$ & $\begin{array}{l}\text { Informe del Departamento de } \\
\text { Atención a la Población }\end{array}$ \\
\hline $\begin{array}{l}\text { Índice de cumplimiento del } \\
\text { esquema de vacunación }\end{array}$ & $\begin{array}{l}\text { Total de dosis aplicadas entre el total de dosis planificadas, expresado en por } \\
\text { ciento }\end{array}$ & $\begin{array}{l}\text { Registro del Departamento de } \\
\text { Vacunación }\end{array}$ \\
\hline $\begin{array}{l}\text { Índice de casos de tuberculosis } \\
\text { diagnosticados en la policlínica }\end{array}$ & $\begin{array}{l}\text { Total de nuevos casos de tuberculosis diagnosticados mediante la prueba del } \\
\text { bacilo resistente al alcohol ácido (BARR) por la policlínica entre el total de } \\
\text { casos diagnosticados (BARR+) en el área de salud, expresado en por ciento }\end{array}$ & $\begin{array}{l}\text { Registro del Programa de Control } \\
\text { de la Tuberculosis }\end{array}$ \\
\hline \multicolumn{3}{|l|}{ De recursos } \\
\hline Enfermeros de familia & Número de enfermeros de familia en los consultorios médicos & Registro de recursos humanos \\
\hline Enfermeros de vacunatorios & Número de enfermeros de vacunatorio en la policlínica & Registro de recursos humanos \\
\hline $\begin{array}{l}\text { Gasto en salarios en la } \\
\text { policlínica }\end{array}$ & $\begin{array}{l}\text { Fondos asignados a la policlínica para el pago de salarios (más } 9,09 \% \text { de esa } \\
\text { cantidad acumulado por concepto de vacaciones), expresado en pesos cubanos }{ }^{a}\end{array}$ & Registros contables \\
\hline Gasto en medicamentos & $\begin{array}{l}\text { Fondos asignados a la policlínica para gastos en medicamentos y reactivos de } \\
\text { laboratorio, expresado en pesos cubanos }\end{array}$ & Registros contables \\
\hline
\end{tabular}

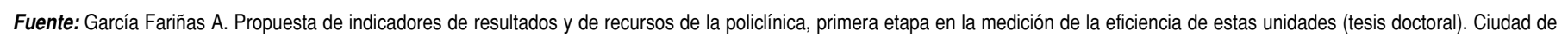
La Habana: Instituto Nacional de Higiene, Epidemiología y Microbiología; 2005.

a Tasa de cambio oficial del Ministerio de Finazas y Precios de la República de Cuba: 1,00 peso cubano =1,00 peso cubano convertible $=1,25$ dólar estadounidense.

Se asumió que habría un comportamiento de rendimientos constantes a escala (RCE), debido a la homogeneidad observada entre las unidades estudiadas (16). Además de que las unidades comparadas están agrupadas en conjuntos de tamaños y características similares, todas forman parte de un sistema de salud único, lo que hace que las características organizativas, de asignación de recursos y de funcionamiento sean similares. Con RCE se tiende a subestimar la eficiencia (15), por lo que se comprobó la pertinencia de este enfoque mediante el cálculo del coeficiente de correlación de Spearman $(r s)$ como medida de la asociación del gasto en salarios - como mejor aproximación del tamaño de la unidad - y el índice de eficiencia productiva calculado. Cuando el valor de $r s$ resultaba mayor que 0,7 se calculó el índice de eficiencia productiva bajo el supuesto de rendimientos variables a escala (RVE), ya que la elevada correlación indicaba que no era válido emplear la premisa de RCE.

Los índices de eficiencia se estimaron mediante el software Frontier Analyst v. 3, específico para este tipo de estudios (17). Para el cálculo de los coeficientes de correlación se utilizó el paquete estadístico SPSS con un nivel de significación de 95\% (18).

\section{RESULTADOS}

La eficiencia promedio de las 40 unidades evaluadas en este estudio fue de $0,95 \pm 0,11$ y $11(27,5 \%)$ de ellas presentaron un desempeño ineficiente de $0,77 \pm 0,12$. En general, las principales reservas para mejorar la eficiencia de las policlínicas de Matanzas fueron aumentar la tasa de detección de los casos de tuberculosis, reducir la mortalidad infantil evitable y ampliar la cobertura de vacunación.

\section{Policlínicas del subgrupo 1A}

En el análisis de correlaciones entre las variables de recursos se obtuvo una fuerte asociación significativa entre la presencia de enfermero de vacunatorio y el resto de las variables de recursos ( $r s=0,78 ; P=0,007)$ por lo que esa variable no se utilizó para el cálculo de la eficiencia en este grupo de policlínicas. También se observó una fuerte asociación entre los índices de satisfacción de la población y de bajo peso al nacer ( $r s=0,67 ; P \leq 0,024$ ), por lo que en este caso sólo se incorporó el índice de bajo peso al nacer al modelo. En este grupo, no se encontró asociación significativa entre la eficiencia y el 
CUADRO 2. Índice de eficiencia, según los subgrupos de policlínicas. Matanzas, Cuba, enero-junio de 2006

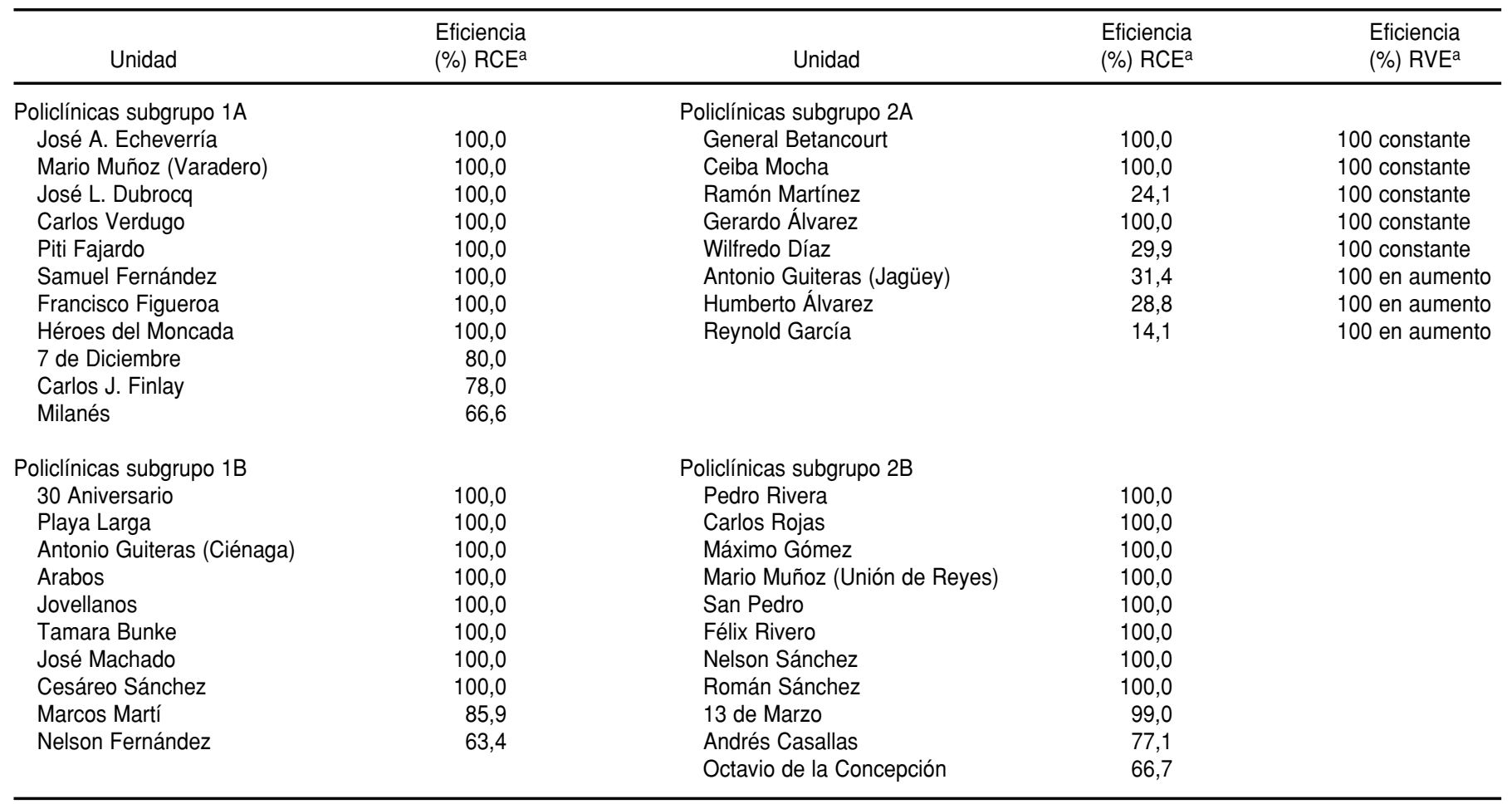

${ }^{a}$ RCE: rendimientos constantes a escala; RVE: rendimientos variables a escala, válido solamente en el grupo $1 \mathrm{~A}$.

gasto en salarios (como variable representativa del tamaño de la unidad) por lo que el supuesto de RCE fue válido.

En el cuadro 2 se muestran los valores de eficiencia relativa de cada policlínica de este subgrupo, tanto bajo el modelo de maximización de resultados como de RCE. Sólo tres unidades ( $27,3 \%$ del total de este grupo) resultaron ineficientes. El análisis de los pares de referencias permitió identificar a las policlínicas Mario Muñoz (Varadero) y José L. Dubrocq como las de mejor práctica y sirvieron de referencia para delimitar las unidades ineficientes (cuadro 3).

Las tres unidades ineficientes en este subgrupo contaban con reservas para elevar los indicadores de resultados. Las reservas de cada policlínica para incrementar la eficiencia fueron muy similares entre sí, a pesar de los diferentes niveles de eficiencia alcanzados por cada una de ellas. Debe señalarse que el valor teórico que debían alcanzar estas policlínicas en las variables inmunización del escolar, índice de bajo peso al nacer y mortalidad infantil evitable, para poder lograr una relación recursos-resultados similar a la del umbral de eficiencia era mayor de $100 \%$ (cuadro 4). Este resultado, aparentemente contradictorio, indica que había un exceso de recursos en esas unidades, como puede verse en el cuadro 5. En este subgrupo de policlínicas se observó un sobredimensionamiento en dos de los cuatro indicadores empleados (enfermero de familia y médico de familia), lo que muestra que según estos indicadores, esas unidades cuentan con más recursos de los necesarios para maximizar sus resultados (cuadro 5).

\section{Policlínicas del subgrupo 1B}

Según los valores del $r s$ entre las variables de resultados, la mortalidad in- fantil evitable está fuertemente asociada con el resto de las variables $(r s=$ $0,78 ; P=0,020)$ por lo que no se incorporó al modelo. De las variables de recursos se encontró que la presencia de enfermeros de vacunatorio estaba fuertemente asociada con el resto de los indicadores $(r s=0,66 ; P=0,007)$. Se encontró una fuerte asociación entre las variables médico de familia y enfermero de familia $(r s=0,79 ; P=0,007)$, así como entre especialista en ginecoobstetricia y gasto en salarios ( $r s=0,67$; $P \leq 0,05)$. Como consecuencia de estos resultados, no se incorporaron en el modelo para este subgrupo los indicadores de recursos enfermero de vacunatorio, médico de familia y gasto en salarios.

Según el modelo de maximización de resultados y de RCE, sólo dos policlínicas $(20,0 \%)$ resultaron ineficientes (cuadro 2). En este subgrupo también se utilizó el supuesto de RCE, ya que no se encontró asociación significativa 
CUADRO 3. Policlínicas eficientes, según los subgrupos de policlínicas evaluadas y el número de unidades para las que sirvieron de referencia (n). Matanzas, Cuba, enero-junio de 2006

\begin{tabular}{|c|c|c|c|c|c|}
\hline Unidad & $n$ & $\%^{b}$ & Unidad & $n$ & $\%^{\mathrm{b}}$ \\
\hline Policlínicas subgrupo $1 \mathrm{~A}$ & & & Policlínicas subgrupo $2 \mathrm{~A}$ & & \\
\hline José A. Echeverría & 1 & 33,0 & General Betancourt & 2 & 66,0 \\
\hline Mario Muñoz (Varadero) & 3 & 100,0 & Ceiba Mocha & 3 & 100,0 \\
\hline José L. Dubrocq & 3 & 100,0 & Ramón Martínez & 1 & 33,0 \\
\hline Carlos Verdugo & 1 & 33,0 & Gerardo Álvarez & 0 & 0,0 \\
\hline Piti Fajardo & 0 & 0,0 & Wilfredo Díaz & 0 & 0,0 \\
\hline Samuel Fernández & 1 & 33,0 & & & \\
\hline Francisco Figueroa & 1 & 33,0 & & & \\
\hline Héroes del Moncada & 1 & 33,0 & & & \\
\hline Policlínicas subgrupo 1B & & & Policlínicas subgrupo 2B & & \\
\hline 30 aniversario & 0 & 0,0 & Pedro Rivera & 2 & 66,0 \\
\hline Playa Larga & 0 & 0,0 & Carlos Rojas & 1 & 33,0 \\
\hline Antonio Guiteras (Ciénaga) & 2 & 100,0 & Máximo Gómez & 2 & 66,0 \\
\hline Arabos & 1 & 50,0 & Mario Muñoz (Unión de Reyes) & 0 & 0,0 \\
\hline Jovellanos & 0 & 0,0 & San Pedro & 0 & 0,0 \\
\hline Tamara Bunke & 1 & 50,0 & Félix Rivero & 1 & 33,0 \\
\hline José Machado & 0 & 0,0 & Nelson Sánchez & 1 & 33,0 \\
\hline Cesáreo Sánchez & 2 & 100,0 & Román Sánchez & 2 & 66,0 \\
\hline
\end{tabular}

${ }^{\mathrm{b}}$ Porcentaje con respecto al total de policlínicas ineficientes en cada subgrupo.

CUADRO 4. Reservas para mejorar la eficiencia en indicadores de resultados en las unidades ineficientes, según los subgrupos de policlínicas. Matanzas, Cuba, enero-junio de 2006

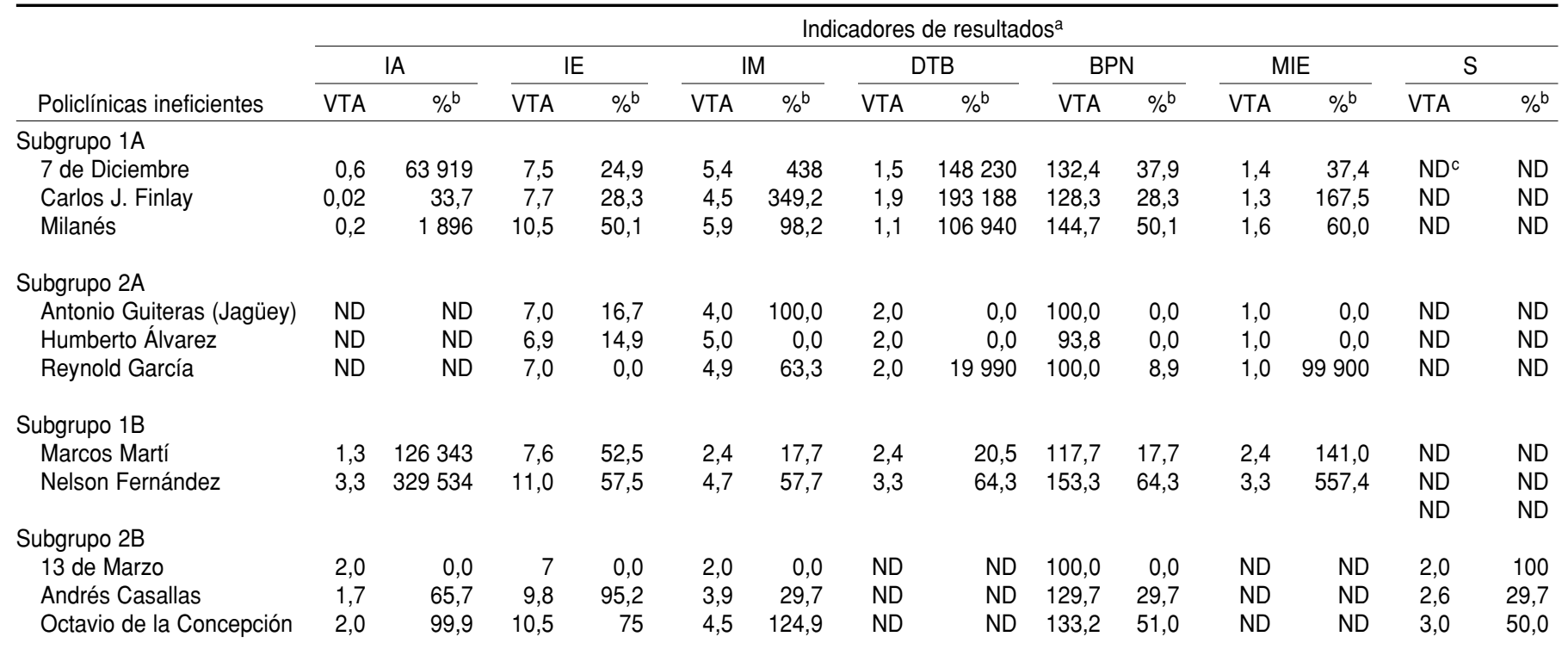

a IA: inmunización de adultos; IE: inmunización de escolares; IM: inmunización de menores de dos años; DTB: detección de tuberculosis; BPN: índice de bajo peso al nacer; MIE: mortalidad infantil evitable; S: índice de satisfacción de la población; VTA: valor teórico a alcanzar.

${ }^{b}$ Porcentaje del incremento respecto al valor actual para alcanzar el VTA.

${ }^{c} \mathrm{ND}=$ no disponible.

entre las variables eficiencia y gasto en salarios.

Las policlínicas de mejor práctica en este subgrupo fueron Antonio Guiteras (Ciénaga) y Cesáreo Sánchez, que se utilizaron como referencia para las dos policlínicas ineficientes (cuadro 3).

Se observó que las policlínicas Marcos Martí y Nelson Fernández contaban con reservas para incrementar su eficiencia en todos los indicadores de resultados. Sin embargo, los valores que deberían alcanzar para ubicarse en la frontera de eficiencia en los cinco indicadores son tan elevados que en la 
CUADRO 5. Reservas para mejorar la eficiencia en indicadores de recursos, según las unidades ineficientes. Matanzas, Cuba, enerojunio de 2006

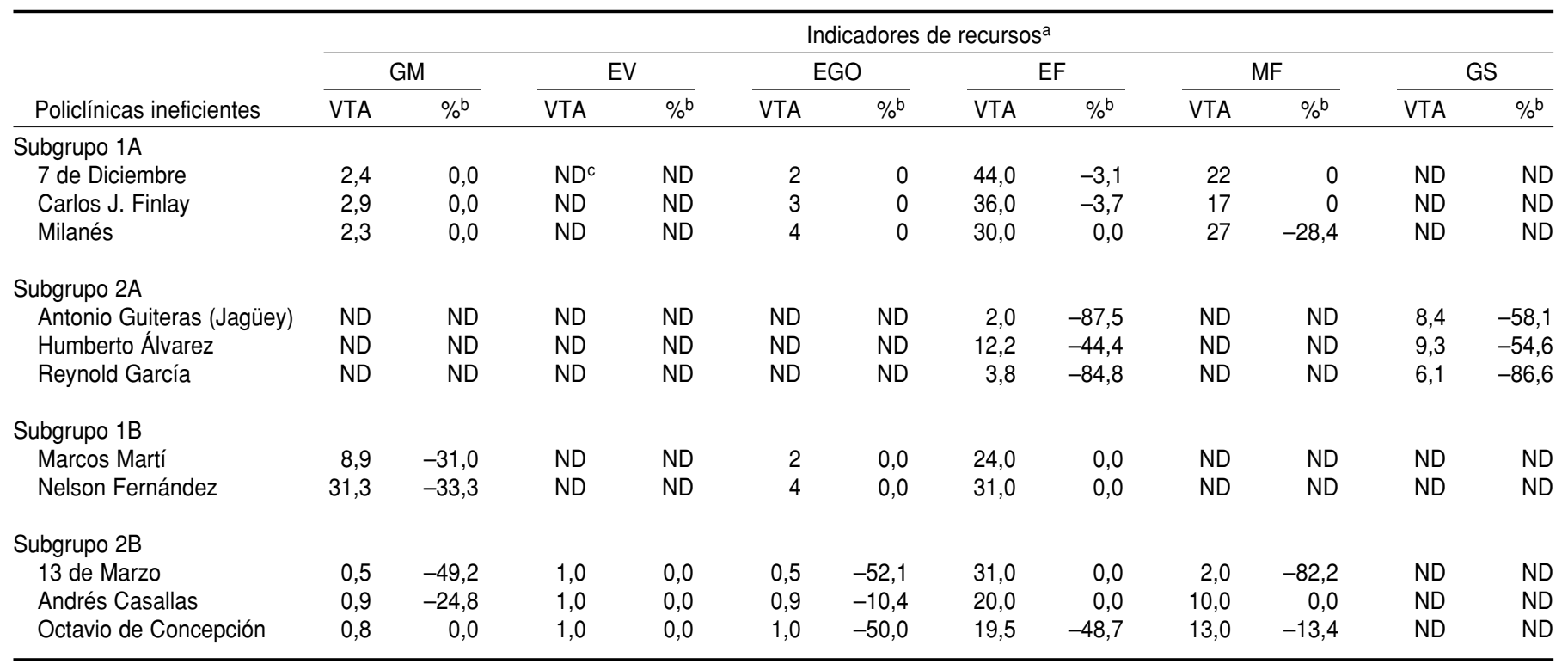

a GM: gasto en medicamento; EV: enfermeros de vacunatorio; EGO: especialistas en ginecoobstetricia; EF: enfermeros de familia; MF: médicos de familia; GS: gasto en salarios; VTA: valor teórico a alcanzar.

${ }^{b}$ Porcentaje del incremento respecto al valor actual para alcanzar el VTA.

${ }^{c} \mathrm{ND}=$ no disponible.

práctica quedan fuera de sus posibilidades, ya que, por ejemplo, deberían alcanzar niveles de coberturas de inmunización mayores de 100\%. De esta forma, aunque estas unidades lograran el máximo en todos los indicadores de resultados, no lograrían alcanzar la eficiencia del resto de las unidades. Como en el subgrupo anterior, estos resultados indican que hay un exceso de recursos, reflejado también en el elevado gasto en medicamentos de estas unidades (cuadro 5), mayor del necesario para maximizar sus resultados. La reducción de este gasto puede ser una reserva potencial para incrementar la eficiencia en estas unidades.

\section{Policlínicas del subgrupo 2A}

En este subgrupo, se encontró una fuerte asociación entre las variables de resultados inmunización del adulto y bajo peso al nacer $(r s=0,75 ; P=0,032)$, por un lado, y los índices de satisfacción de la población y de casos de tuberculosis diagnosticados en la policlí- nica $(r s=0,96 ; P=0,023)$ por el otro. A partir de estos resultados se decidió tomar solamente una variable de cada par: los índices de bajo peso al nacer y de detección de casos de tuberculosis.

Se encontraron tres pares de variables de recursos con una fuerte asociación entre la presencia de enfermero de familia y de especialista en ginecoobstetricia $(r s=0,85 ; P=0,008)$; enfermero de familia y médico de familia ( $r s=0,82 ; P=0,013)$; y especialista en ginecoobstetricia y gasto en medicamentos ( $r s=0,83 ; P \leq 0,011)$. Al igual que con los indicadores de resultados, sólo se tomó una de las variables de cada par, por lo que se incorporaron al modelo las variables presencia de enfermero de familia y gasto en medicamentos.

Los valores de eficiencia obtenidos según el modelo de RCE muestran que cinco policlínicas $(62,5 \%)$ fueron ineficientes. Sin embargo, se encontró una fuerte asociación $(r s=0,95 ; P=$ 0,021 ) entre el índice de eficiencia y el tamaño de la unidad (representado por el gasto en salarios), por lo que se recalculó el índice de eficiencia según el supuesto de RVE (cuadro 2). Se encontró que, excepto en las policlínicas General Betancourt, Ceiba Mocha y Gerardo Álvarez, en todas las unidades aumentaron los valores de eficiencia y todas las policlínicas de este subgrupo resultaron eficientes. No obstante, las unidades Humberto Álvarez, Reynold García y Antonio Guiteras (Jagüey) no alcanzaron el nivel de productividad máximo y pueden mejorar su eficiencia.

La policlínica Ceiba Mocha tuvo los mejores resultados dentro de este subgrupo y sirvió de referencia para las unidades ineficientes.

Las reservas de las policlínicas Humberto Álvarez, Reynold García y Antonio Guiteras (Jagüey) en al menos uno de los indicadores de resultados les pueden permitir alcanzar niveles de eficiencia con valores teóricos ubicados dentro de los márgenes alcanzables. El indicador de resultado con menos reservas fue el índice de bajo peso al nacer (cuadro 4). No obstante, también en este subgrupo algunas variables de recursos están sobredimensionadas, por lo que su reducción tam- 
bién constituye una reserva para mejorar la eficiencia (cuadro 5).

\section{Policlínicas del subgrupo 2B}

En este subgrupo se encontró una fuerte asociación entre los indicadores de recursos especialista en ginecoobstetricia y gasto en salarios $(r s=0,75 ; P=$ $0,008)$, por lo que solamente se incorporó el indicador de la presencia de especialista en ginecoobstetricia al modelo para calcular el índice de eficiencia. Entre los indicadores de resultados se encontró que el índice de casos de tuberculosis diagnosticados en la policlínica $(r s=0,86 ; P=0,008)$ y la mortalidad infantil evitable $(r s=0,82 ; P=$ $0,012)$ se asociaron fuertemente con el resto de los indicadores, por lo que ninguno de los dos se incorporó al modelo de cálculo de la eficiencia.

Según los valores de eficiencia obtenidos para cada policlínica, tres unidades $(27,3 \%)$ resultaron ineficientes (cuadro 2). No se observó asociación significativa entre la variable eficiencia - calculada bajo el supuesto de RCE (cuadro 1) - y el gasto en salarios (como variable representativa del tamaño de la unidad), por lo que se aceptó que tenían un comportamiento de RCE.

En este subgrupo resultó difícil seleccionar una unidad de referencia con mejor práctica, por lo que todas las policlínicas eficientes se utilizaron como referencia para mejorar la eficiencia de las policlínicas ineficientes (cuadro 3 ).

La policlínica 13 de Marzo mostró un comportamiento singular, ya que sus reservas estaban concentradas en un solo indicador de resultados (el índice de satisfacción de la población) y tres de los cinco indicadores de recursos mostraron que había holgura de recursos (cuadro 4). Las reservas potenciales de las unidades Andrés Casallas y Octavio de la Concepción en los indicadores de resultados eran limitadas, debido a que en la práctica no es posible alcanzar el valor teórico propuesto. A esto se debe añadir que estas policlínicas presentan holgura de recursos en al menos dos de los cinco indicadores de recurso empleados.

\section{DISCUSIÓN}

Un aspecto fundamental en la evaluación de la gestión de las organizaciones sanitarias es la capacidad de identificar las organizaciones que tienen una gestión adecuada o deficiente en relación con alguna referencia. En el presente trabajo se caracterizó la eficiencia productiva de las policlínicas de la provincia de Matanzas, Cuba. Esta información, que se publica por primera vez, debe contribuir a optimizar la eficiencia de las instituciones de APS y constituye una herramienta muy útil para mejorar la gestión general de estas unidades.

En la provincia de Matanzas, entre $60 \%$ y $80 \%$ de las policlínicas se clasificaron como eficientes, a pesar de que algunos autores señalan que solamente alrededor de un tercio de las unidades alcanzan esa clasificación. Esto podría deberse al poco poder discriminatorio de las técnicas empleadas o a que estos resultados pueden estar afectados por la relación entre el número de variables incluidas y la cantidad de unidades evaluadas: cuanto menor es el número de variables y mayor es el tamaño de la muestra, mayor es el poder discriminatorio de la técnica (14). Pudiera pensarse, además, en un posible efecto de práctica homogénea en la provincia estudiada. Como el AED es una técnica que estima valores relativos, la similitud entre las unidades - tanto en términos de resultados alcanzados como de recursos disponibles- puede llevar a que muestren niveles similares de eficiencia, aun cuando la proporción entre la cantidad de variables y el tamaño de la muestra sea la recomendada.

En 7 de los 14 municipios de la provincia de Matanzas (Varadero, Jovellanos, Perico, Los Arabos, Calimete, Ciénaga y Pedro Betancourt), todas las policlínicas se clasificaron como eficientes. Los municipios con mayores deficiencias fueron Martí y Limonar, con todas sus policlínicas ineficientes, seguidos de Jagüey, que tenía $66 \%$ de sus unidades ineficientes. Sin embargo, esto puede deberse a debilidades gerenciales - como deficiencias en la planificación, la organización, la dirección y el control de los recursos hu- manos y el insuficiente seguimiento de los resultados-, el inadecuado seguimiento de los grupos de población más vulnerables, la deficiente preparación de los recursos humanos y la falta de participación de la comunidad en las acciones de salud desarrolladas en el área.

En todas las policlínicas del tipo 1 (de mayor complejidad) y en las del tipo 2A (de menor complejidad ubicadas en los municipios de mayor nivel socioeconómico), las mejoras potenciales o reservas de eficiencia se concentraron en la inmunización de adultos y en la detección de casos de tuberculosis. Por tanto, las acciones se deben concentrar en estás direcciones si se quiere mejorar la eficiencia de estas unidades. Sin embargo, las policlínicas del tipo 2B (de menor complejidad y ubicadas en los municipios de menor desarrollo) mostraron una mayor diversidad en sus reservas de eficiencia, lo que puede indicar que se necesitan intervenciones más integrales para mejorar la eficiencia en esas unidades.

La eficiencia promedio de las unidades evaluadas en este estudio fue de $0,95 \pm 0,11$, similar a las reportadas para unidades de APS en países de Europa $(0,82 \pm 0,12)$ y superior a la informada en unidades estadounidenses $(0,648 \pm 0,25)(5)$.

En general, 27,5\% de las policlínicas estudiadas tuvieron un desempeño ineficiente $(0,77 \pm 0,12)$, no obstante, su nivel de eficiencia relativa fue alto en comparación con lo informado en centros de salud africanos. De 17 centros de salud de Ghana, 3 (18\%) tenían un desempeño valorado de deficiente $(0,49 \pm 0,27)(19)$, mientras que $59 \%$ de las unidades de salud evaluadas en Sierra Leona se clasificaron como ineficientes (15). No obstante, este tipo de comparaciones se debe hacer con suma cautela, pues los diseños de investigación elegidos pueden diferir.

En relación con las variables de resultados, no son frecuentes los estudios con diseños similares al empleado en el presente trabajo. Por ejemplo, de los 188 estudios revisados por Hollingsworth (5), solamente en 10 se emplearon medidas de resultados. Otros autores han empleado como variables 
de resultados algunos indicadores de proceso, como días de ingreso o número de consultas $(5,19)$.

En las 11 policlínicas ineficientes de Matanzas se encontraron evidencias del llamado slakc u holgura de recursos en al menos un indicador. Los especialistas en ginecoobstetricia constituyeron el recurso que más veces mostró este comportamiento, con una frecuencia de $54,5 \%$, seguido del gasto en medicamentos en 4 de las 11 unidades $(36,4 \%)$. La holgura en los indicadores de recursos indica que las unidades contaban con más recursos de los que necesitaban para maximizar sus resultados. Para poder llegar a esta conclusión, debe haber otras policlínicas con menos recursos y resultados similares o mejores dentro del subgrupo analizado.

En este estudio se asumió que en el proceso productivo había una relación directa entre la cantidad de recursos y la cantidad de resultados en un período de tiempo dado. Sin embargo, como el resultado se valoró mediante indicadores de salud, la totalidad de los recursos que participaron en el logro de esos resultados no tienen necesariamente que corresponderse con los recursos existentes en el momento de la medición. Es decir, el resultado que se mide en un momento dado puede estar relacionado con el trabajo realizado durante un periodo de tiempo anterior y con un conjunto diferente de recursos. Así, el índice de bajo peso al nacer medido en las policlínicas durante el primer trimestre del año no es únicamente el resultado de las acciones y de los recursos de la policlínica en ese trimestre, sino el resultado acumulado de varios meses de trabajo anteriores, cuando el nivel de recursos pudo ser diferente. Estudios posteriores mediante series cronológicas permitirán aclarar si con mucho menos recursos se pueden lograr los resultados que espera el SNS. No obstante, la evidencia aportada por esta investigación puede contribuir a mejorar el proceso de asignación de los recursos.

Entre las limitaciones del presente estudio está el no contar con información sobre el comportamiento de la eficiencia durante el resto de los trimestres del año en las unidades analizadas. Aun así, la información presentada demuestra la posibilidad de realizar este tipo de estudios a partir de los datos del registro continuo que se lleva en el SNS de Cuba y constituye una nueva referencia metodológica para el análisis del resto de los trimestres. Además, estos resultados deben contribuir a lograr una mejor gestión de los recursos públicos.

A manera de conclusiones, se puede señalar que las policlínicas de la provincia de Matanzas muestran un nivel elevado de eficiencia. En todos los subgrupos de policlínicas evaluadas se identificaron dos o más unidades de referencia, lo que permitió identificar elementos organizacionales que podrían contribuir a mejorar la eficiencia de las unidades deficientes. Las principales reservas para aumentar la eficiencia fueron aumentar la detección de los casos de tuberculosis en la comunidad, reducir el índice de mortalidad infantil evitable y ampliar la cobertura de inmunización. En las 11 policlínicas ineficientes se observó holgura de recursos en al menos un indicador. Se debe revisar el proceso de asignación de los recursos a las policlínicas del SNS para evitar el exceso de recursos innecesarios como vía para aumentar la eficiencia.

\section{REFERENCIAS}

1. Palmer S, Torgerson DJ. Definitions of efficiency. Br Med J. 1999;318:1136.

2. Drummond M, Stoddart G, Torrance G. Método para la evaluación económica de los programas de atención de salud. Madrid: Ediciones Díaz de Santos; 1991.

3. Puig-Junoy J, Matarrodono E. ¿Qué sabemos acerca de la eficiencia de las organizaciones sanitarias en España? Una revisión de la literatura económica. XX Jornadas de Economía de la Salud; 2000 mayo 3-5; Palma de Mallorca, España. Palma de Mallorca: Asociación de Economía de la Salud; 2000. Hallado en: http:// www.econ.upf.edu/ puig/publicacions/ paper999.pdf. Acceso el 2 de octubre de 2006.

4. Pinillos M, Antoñanzas F. La atención primaria de salud: descentralización y eficiencia. Gac Sanit. 2002;16(5):401-7.

5. Hollingsworth B. Non-parametric and parametric applications measuring efficiency in health care. Health Care Manag Sci. 2003;6: 203-18.
6. Trick M. Data envelopment analysis for consultants [sitio en Internet]. Hallado en: http:// mat.gsia.cmu.edu/mstc/dea/dea.html. Acceso el 13 de enero de 2004.

7. Farrell MJ. The measurement of productive efficiency. J R Stat Soc Ser A General. 1957; 120:252-90.

8. Charnes A, Cooper WW, Rhodes E. Measuring the efficiency of decision making units. Eur J Oper Res. 1978;2:429-44.

9. Gattoufi S, Oral M, Kumar A, Reisman A. Epistemology of data envelopment analysis and comparison with other fields of OR/MS for relevance to applications. Socioecon Plann Sci. 2004;38:123-40.

10. Gattoufi S, Oral M, Reisman A. A taxonomy for data envelopment analysis. Socioecon Plann Sci. 2004;38:141-58.

11. Gálvez AM. El concepto de eficiencia en el contexto de la salud pública cubana. La Habana: Escuela Nacional de Salud Pública; 2002.
12. Organización Panamericana de la Salud, Organización Mundial de la Salud. Eficiencia en la atención primaria de salud. La Habana: Organización Panamericana de la Salud; 2006. (Serie Desarrollo No. 22.A).

13. Valdivia Onega NC. Epidemiología y atención primaria de salud. Rev Cubana Hig Epidemiol [publicación periódica en línea]. 2003;41(2-3). Hallado en: http://scielo.sld. $\mathrm{cu} /$ scielo.php?script=sci_arttext\&pid=S1561$30032003000200010 \& \operatorname{lng}=e s \& n r m=i s o$. Acceso el 5 de septiembre de 2007.

14. Rojas Ochoa F. La atención primaria de salud en Cuba, 1959-1984. Rev Cubana Salud Publica [publicación periódica en línea]. 2005; 31(2). Hallado en: http:/ /scielo.sld.cu/scielo. php? script $=$ sci_arttext\&pid $=S 0864$ $34662005000200011 \& \operatorname{lng}=\mathrm{es} \& n r m=i s o \& t \operatorname{lng}=$ es. Acceso el 5 de septiembre de 2007.

15. Renner A, Kirigia JM, Zere EA, Barry SP, Kirigia DG, Kamara Cand Muthuri LHK. Technical efficiency of peripheral health units in $\mathrm{Pu}$ - 
jehun district of Sierra Leone: a DEA application. BMC Health Serv Res. 2005;5:77.

16. Banker RD, Charners A, Cooper WW. Some models for estimating technical and scale inefficiencies in data envelopment analysis. Manage Sci. 1984;30(2):1078-92.

17. Frontier Analyst. Free demonstration software. Cumbria, Reino Unido: Frontier Ana- lyst; 2004. Hallado en http://www.banxia. com/frontier/index.html. Acceso el 31 de julio de 2007.

18. SPSS Base 10.0 for Windows. User's Guide. Chicago, IL: SPSS Inc., 1999.

19. Osei D, d'Almeida S, George MO, Kirigia JM, Mensah AO, Kainyu LH. Technical efficiency of public district hospitals and health centres in Ghana: a pilot study. Cost Eff Resour Alloc. 2005;27(3):9.

Manuscrito recibido el 25 de octubre de 2006. Aceptado para publicación, tras revisión, el 26 de junio de 2007.

ABSTRACT Objectives. To rate the efficiency of all the outpatient clinics in Matanzas, Cuba; identify the best-performing clinics; and find opportunities for improvement at the others.

\section{Efficiency of outpatient clinics in Matanzas, Cuba, using data envelopment analysis}

Methods. A descriptive study of the 40 outpatient clinics in the province of Matanza was carried out during the first trimester of 2006. Clinics were grouped according to the complexity of services they offer and the socioeconomic level of the municipality in which they are located. Five output and six input variables were analyzed. Calculations were performed using data envelopment analysis, including optimization of results and constant and variable returns-to-scale.

Results. In general, the clinics studied had high efficiency rates, with a mean of $0.95 \pm 0.11$. Eleven $(27.5 \%)$ clinics studied were rated inefficient $(0.77 \pm 0.12)$. The following Three key areas for improvement were identified: increasing tuberculosis detection rates in the community, reducing rates of preventable infant mortality, and expanding immunization coverage. Among the 11 clinics rated as inefficient, there were resource gaps in at least one indicator.

Conclusions. By recognizing successful clinics, best practices were identified that could be used to improve the weaker clinics. The resource distribution process should be reviewed to ensure that additional, unneeded resources are not used to improve efficiency.

Key words Health services research; efficiency, organizational; primary health care, Cuba.

\section{Immunization Field Guides Set*}

This set of six Field Guides will help health workers in their efforts to control, eliminate, or eradicate diseases such as poliomyelitis, neonatal tetanus, yellow fever, diphtheria, pertussis, tetanus, Haemophilus influenzae type b infections, hepatitis B, measles, and rubella. The Field Guides also include standardized methods and procedures for conducting epidemiological surveillance and maintaining an up-to-date information system that makes it possible to make timely, effective decisions.

*The six Field Guides in the set are:

(1) Control of Yellow Fever: Field Guide

(2) Measles Elimination: Field Guide

(3) Polio Eradication: Field Guide

(4) Elimination of Neonatal Tetanus: Field Guide

(5) Control of Diphtheria, Tetanus, Pertussis, Haemophilus influenzae type b (HIB), and Hepatitis B: Field Guide

**(6) Elimination of Rubella and Congenital Rubella Syndrome: Field Guide

* This set can be purchased only through the PAHO Online Bookstore: http://publications.paho.org.

** The Elimination of Rubella and Congenital Rubella Syndrome: Field Guide will be published in 2008.

We will send that Field Guide to you separately, as soon as it is published.

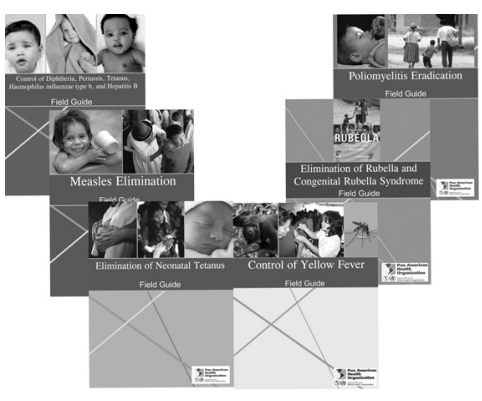

ISBN: 9275126860 Order Code: ST 057 Price: US\$ 36.00 in Latin America and the Caribbean/ $\$ 48.00$ elsewhere 